\title{
Das Berufsbild wissenschaftlicher Bibliothekarinnen und Bibliothekare im Wandel
}

\author{
Perspektiven aus der Schweiz und aus Deutschland
}

\begin{abstract}
„Wer bin ich - und wenn ja, wie viele? Für wen? Und wie lange noch?“ Das mag sich in Anlehnung an den bekannten Buchtitel von Richard David Precht so manche wissenschaftliche Bibliothekarin und so mancher wissenschaftliche Bibliothekar ${ }^{1}$ fragen.

Aktuelle Stellenanzeigen für wissenschaftliches Bibliothekspersonal ${ }^{2}$ zeigen ein beachtliches Spektrum von Tätigkeiten: Einsatz in Forschung und Entwicklung oder als Fachbereichs-Netzwerker, im Bestandsmanagement oder in der Altbestandspflege, bei der Entwicklung neuer E-Services oder im Bereich E-Learning, Stabsstellen für IT-Dienste oder für Aus- und Fortbildung, Qualitätsmanagement oder Bibliometrie, Abteilungsleitungen oder Forschungsdatenmanagement u. v. m.

Nur: Was haben der Leiter einer Handschriftenabteilung in einer Landesbibliothek, die stellvertretende Leiterin einer Großstadtbibliothek, ein Dozent an einer bibliothekarischen Ausbildungseinrichtung, eine Projektmitarbeiterin im Forschungsdatenmanagement, eine Fachreferentin für Medizin oder eine Stabsstellenleiterin E-Resource and Metadata Management gemeinsam? ${ }^{3}$ Ist wissenschaftliche Bibliothekarin oder wissenschaftlicher Bibliothekar überhaupt (noch) ein Beruf mit definiertem Berufsbild in Bezug auf Ausbildung, Tätigkeitsmerkmale und Anforderungen des Arbeitsmarktes?

In Zeiten immer schnellerer Veränderung lassen sich diese und weitere Fragen je nach Standpunkt und Blickwinkel nicht leicht und schon gar nicht eindeutig
\end{abstract}

1 Die Verf. erläutern: „Abweichend von der Regelung für den vorliegenden Band wurde dieser Text nach den Prinzipien der sprachlichen Gleichstellung von Frauen und Männern verfasst. Eine Diskriminierung von Personen mit anderer Geschlechtsidentität ist dadurch nicht beabsichtigt.“

2 Für eine empirische Analyse von Stellenzeigen der Jahre 2003-2013 vgl. Braun \& Brunenberg-Piel 2014.

3 Ähnlich fragte bereits Schibel 1998.

DOI 10.1515/9783110481037-025 
beantworten. Trotzdem oder gerade deswegen greift sie der vorliegende Beitrag auf und beleuchtet sie aus Schweizer Perspektive mit einigen Seitenblicken auf Deutschland. ${ }^{4}$

\section{Der Beruf und sein Berufsbild}

Nach dem populären Online-Nachschlagewerk Wikipedia ist ein Beruf die ,aufgrund besonderer Eignung und Neigung systematisch erlernte und mit einem Qualifikationsnachweis versehene, dauerhaft und gegen Entgelt ausgeübte spezialisierte Betätigung eines Menschen“5. Der erlernte (Ausbildungs-)Beruf mit einem formalen Qualifikationsnachweis und der tatsächlich ausgeübte (Erwerbs-)Beruf können, müssen aber nicht deckungsgleich sein. In der Alltagssprache kommt dieser Unterschied beispielsweise in den Formulierungen „Ich bin ...“ und „Ich arbeite als ...“ zum Ausdruck.

Ein Berufsbild beschreibt die Elemente eines Berufes wie Vorbildung, Ausbildung, Tätigkeiten, Aufstiegschancen, Weiterbildungsformen und Verdienstmöglichkeiten, und insbesondere bei Ausbildungsberufen auch Lerninhalte, Prüfungen etc. ${ }^{6}$ Ein Berufsbild ist auch das Bild, das sich jemand von einem Beruf macht. ${ }^{7}$ Berufsbilder werden von Berufsverbänden oder anderen Institutionen festgelegt oder durch gesetzliche Regelungen vorgegeben. Sie dienen nicht nur der Abgrenzung von anderen Berufen, der Darstellung eines Berufsfeldes gegenüber potenziellen Berufseinsteigerinnen und -einsteigern, Arbeitgebenden oder Behörden sowie der breiten Öffentlichkeit, sondern auch der eigenen Selbstvergewisserung und -bestätigung derjenigen, die den darin beschriebenen Beruf erlernt haben oder ausüben.

Berufe werden unter anderem nach Branche, Ausbildungsabschluss, Grad der Selbstständigkeit oder sozialem Status unterschieden. National und international existieren dafür verschiedene Systematiken. In der Klassifikation der Berufe der deutschen Bundesagentur für Arbeit zählen Berufe in Bibliotheken zur Kategorie „Unternehmensorganisation, Buchhaltung, Recht und Verwaltung“, Unterkategorie „Medien-, Dokumentations- und Informationsdienste“.

4 Dieser Text basiert auf den Präsentationen der Autorinnen beim Deutschen Bibliothekartag 2015 in Nürnberg.

5 Wikipedia 2016a.

6 Brockhaus Enzyklopädie Online 2015.

7 Munzinger Online 2015. 
Zusätzlich werden sie gegliedert in fachlich ausgerichtete Tätigkeiten, komplexe Spezialistentätigkeiten und hoch komplexe Tätigkeiten. ${ }^{8}$

Zur Kategorie „Berufe im Bibliothekswesen - hoch komplexe Tätigkeiten“ zählen ,alle Berufe im Bibliothekswesen, deren Tätigkeiten einen hohen Komplexitätsgrad aufweisen und ein entsprechend hohes Kenntnis- und Fertigkeitsniveau erfordern. Angehörige dieser Berufe sammeln, wählen, entwickeln, organisieren und pflegen Bibliothekssammlungen und stellen Informationen für Benutzer/innen bereit.“ Ebenfalls werden dieser Berufskategorie zugeschrieben „Aufgaben in der Bibliotheksverwaltung“ und „Forschungstätigkeiten (...), z. B. die Herstellung und Verbreitung von Büchern in handschriftlicher, gedruckter und elektronischer Form in Vergangenheit und Gegenwart erforschen, und wissenschaftliche Arbeiten und Berichte verfassen“. Diese umfasst unter anderem folgende, teilweise synonyme Berufsbenennungen: Beamt(er/in) - wissenschaftliche Bibliotheken (höherer Dienst), Bibliothekar/in - wissenschaftliche Bibliotheken (höherer Dienst), Bibliotheks- und Informationswissenschaftler/in, Bibliotheksbeamt(er/in) (höherer Dienst), Bibliothekswissenschaftler/in, Master of Science - Bibliotheks- u. Informationswissenschaft.

In einer weiteren Kategorie werden die „Führungskräfte - Medien-, Dokumentations- und Informationsdienste“ aufgeführt. Diese ,übernehmen Führungsaufgaben (...), welche einen hohen Komplexitätsgrad aufweisen und ein entsprechend hohes Kenntnis- und Fertigkeitsniveau erfordern“ und sind für „Leitung und Mitarbeiterführung“ verantwortlich. Für diese Kategorie wird die Benennung „Bibliotheksleiter/in“ verwendet. ${ }^{9}$

Die deutsche Übersetzung der International Standard Classification of Occupations (ISCO 08) unterscheidet zwischen „Führungskräfte(n) in der Erbringung von speziellen Dienstleistungen“, die über spezialisierte Qualifikationen und umfassende Erfahrung verfügen und „für Planung, Leitung und Koordinierung der Bereitstellung von speziellen und technischen Dienstleistungen“ unter anderem für Bibliotheken verantwortlich sind, und der Kategorie „Bibliothekswissenschaftler und verwandte Informationswissenschaftler“ in der Hauptgruppe „akademische Berufe“, Untergruppe „Juristen, Sozialwissenschaftler und Kulturberufe“. Für letztere wird ein detaillierter Tätigkeitskatalog aufgelistet. ${ }^{10}$

Etwas konkreter werden die deutschen Berufsverbände. Aus der Fülle der Veröffentlichungen von VDB und BIB sei stellvertretend aus dem Berufsbild Wissenschaftliche/r Bibliothekar/in der BIB-Kommission Ausbildung und Berufsbilder zitiert:

8 Vgl. Statistik der Bundesagentur für Arbeit 2013.

9 Vgl. Statistik der Bundesagentur für Arbeit 2016.

10 Vgl. Statistik Austria 2011. 


\begin{abstract}
Das Einsatzgebiet der wissenschaftlichen Bibliothekare umfasst sowohl fachwissenschaftliche als auch organisatorische Aufgaben. Als Fachreferenten sind sie für die Auswahl und Erschließung der Fachliteratur der verschiedensten Wissenschaftsfächer zuständig sowie für die fachspezifische Benutzerberatung und die Vermittlung von Informationskompetenz. Als Leiter von Organisationseinheiten übernehmen sie managementspezifische Organisations-, Haushalts- und Planungsaufgaben innerhalb der Bibliothek und darüber hinaus in bibliothekarischen Belangen im universitären und regionalen Umfeld. An Hochschulen sind sie Schnittstelle zwischen Bibliothek und wissenschaftlichem Personal. Die Entwicklung neuer Konzepte und Dienstleistungen sowie die Durchführung von Projekten können ebenfalls Bestandteil ihrer Tätigkeit sein. ${ }^{11}$
\end{abstract}

Auf dem Schweizer Berufsberatungsportal ist „Wissenschaftliche/r Bibliothekar/ in“ seit 2013 unter den Hochschulberufen im Berufsfeld Kultur mit einem eigenen Tätigkeitsbeschrieb aufgeführt. ${ }^{12}$

Abgeleitet aus den erwähnten Berufsklassifikationen lässt sich unter der Bezeichnung „wissenschaftliche/r Bibliothekar/in“ ein Verwaltungs-, Kultur- und/ oder Dienstleistungsberuf von hoher Komplexität verstehen, der in der Regel eine akademische Vorbildung voraussetzt und mit anspruchsvollen inhaltlichen und/ oder führungsbezogenen Aufgaben in wissenschaftlichen Bibliotheken verbunden ist. Die Bezeichnung „wissenschaftliche/r Bibliothekar/in“ kommt allerdings weder in der KldB 2010 noch in der ISCO 08 vor. Zudem konzentrieren sich die Berufsklassifikationen auf die Beschreibung von Tätigkeiten und Anforderungsniveaus und geben keine Auskunft darüber, wer innerhalb des Bibliothekswesens tatsächlich als „wissenschaftliche Bibliothekarin“ oder „wissenschaftlicher Bibliothekar" bezeichnet wird bzw. sich selbst als solche/r versteht und auf welcher Basis. Auch die Art der vorausgesetzten (akademischen) Vorbildung und allfälliger bibliothekarischer (Zusatz-)Qualifikationen bleibt offen. Hierzu lohnt sich ein genauerer Blick auf die Ausbildungslandschaft für bibliothekarische Berufe.

\title{
2 Wissenschaftliche Bibliothekarinnen und Bibliothekare in der bibliothekarischen Ausbildungslandschaft
}

Gründe für Änderungen von Berufsbildern, Tätigkeitsfeldern und damit verbunden Ausbildungswegen sind unter anderem äußere, wirtschaftlich-soziale wie

11 BIB 2016.

12 Vgl. Berufsberatung.ch 2016. Seit 2013 auf Anregung der IG WBS und auf der Basis des von dieser erarbeiteten Schweizer Berufsbildes (IG WBS 2013). 
technische Umbrüche. Für die letzten Jahrzehnte sei nur stichworthaft an die Rezeption neuer Managementmethoden (seit ca. Ende der 1980er Jahre das sogenannte neue Steuerungsmodell, später Kosten-Leistungsrechnung oder Anforderungen einer Lernenden Organisation) erinnert sowie an den sich seit langem vollziehenden, aber noch längst nicht abgeschlossenen Wandel, der mit Internet und Digitalisierung einhergeht. Qualifizierung für neue Tätigkeitsfelder, neue technische und organisatorische Mittel, Entwicklung neuer Services für veränderte Nutzungsanforderungen: All dies beeinflusst auch die Ausbildungslandschaft.

In Deutschland lassen sich mindestens drei Hauptlinien für diese Veränderungen der letzten 15 bis 20 Jahre beobachten: Der Beruf der /des Fachangestellten für Medien- und Informationsdienste (FaMI) ab 1998, der Bachelor als grundständiges bibliothekarisches Studium und die Ablösung des Referendariats in den meisten deutschen Bundesländern (ab 2002 etwa in NRW mit Einführung der Masterausbildung, in anderen Bundesländern auch als Volontariat) markieren eine weitgehende Abkehr der vormals tradierten verwaltungs-, d. h. auch bibliotheksinternen Ausbildung. ${ }^{13}$

Die Zugangswege sind vielfältiger und die Durchlässigkeit der Qualifizierungsebenen größer geworden: Berufsbegleitende Aus- oder Weiterbildungen und Fernstudiengänge haben sich etabliert; mit der Ausbildung zur Fachwirtin / zum Fachwirt können sich Fachangestellte weiterqualifizieren, nach einem bibliotheksfachlichen Bachelor-Studiengang kann in konsekutiven Studiengängen ein bibliotheksfachlicher Master angeschlossen werden. Dennoch ist für die Wissenschaftliche Bibliothekarin oder den Wissenschaftlichen Bibliothekar in Deutschland die nicht-konsekutive Studienfolge die Regel, so Konstanze Söllner: „Das traditionelle Berufsbild Wissenschaftlicher Bibliothekare ist nach wie vor durch die Kombination eines universitären Fachstudiums mit Master- oder gleichwertigem Studienabschluss mit einer bibliothekarischen Zusatzausbildung nach dem Muster des Referendariats geprägt."14

Große Berufschancen hat, wer nach dem Fachstudium eine bibliothekarische postgraduale Zusatzqualifikation erwirbt. Dabei setzt der bibliotheksfachliche Master - ob als Fernstudium oder als Weiterbildungs-Master - berufspraktische Erfahrungen voraus. Bibliotheken können für den wissenschaftlichen Dienst entweder jemanden mit abgeschlossener LIS-Zusatzqualifikation einstellen, oder z. B. für die Übernahme von Fachreferaten - eine Person mit der betriebsintern benötigten fachwissenschaftlichen Qualifikation, mit der ggf. eine Vereinbarung

$13 \mathrm{Zu}$ den einzelnen Ausbildungswegen in Deutschland siehe Söllner 2014.

14 Söllner 2014, S. 882. 
über eine $\mathrm{zu}$ absolvierende bibliotheksfachliche Zusatzqualifikation getroffen wird.

In der Schweiz wird die Ausbildung im Bereich Information und Dokumentation (I+D) von den Verbänden aus Archiv, Bibliothek und Informations-/Dokumentationswesen gemeinsam getragen. Sie besteht aus einer Berufslehre mit Eidgenössischem Fähigkeitszeugnis als berufliche Grundbildung und je einem Fachhochschul-Bachelorstudium mit konsekutivem Masterstudiengang in der deutschen und der französischsprachigen Schweiz sowie verschiedenen Angeboten zur Weiterbildung und Weiterqualifizierung auf Tertiärstufe (vgl. Abb. 1).

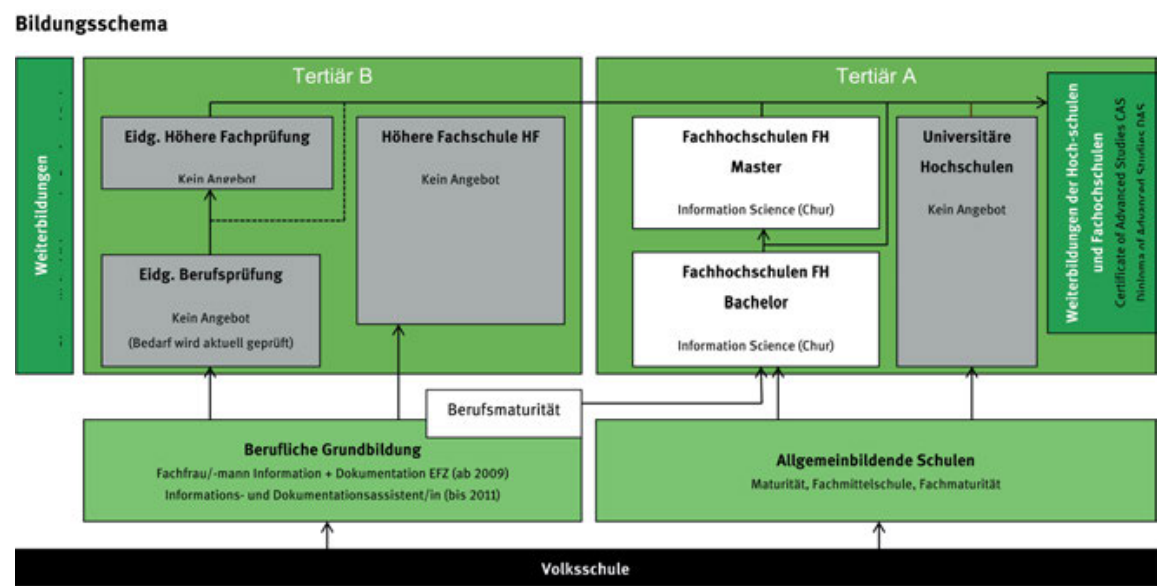

Abb. 1: Bildungsschema I+D-Bereich Schweiz (Ausbildungsdelegation 2016a). ${ }^{15}$

Berufslehre und Fachhochschul-Bachelorstudium lösten Ende der 1990er Jahre die bis dahin bestehende, vom nationalen bibliothekarischen Berufsverband ${ }^{16}$ getragene Ausbildung als Diplombibliothekar bzw. Diplombibliothekarin ab.

Nicht ersichtlich in Abb. 1 ist der Zugangsweg zum Berufsfeld ,wissenschaftliche Bibliothekarin / wissenschaftlicher Bibliothekar", der in der Regel aus einem Hochschulstudium mit universitärem Masterabschluss oder Promotion, seltener mit einem universitärem Bachelorabschluss oder einem FH-Abschluss, sowie einem postgradualen Master of Advanced Studies (MAS)-Studiengang in Archiv-, Bibliotheks- und Informationswissenschaften besteht (vgl. Abb. 2 und Tabelle 1).

15 Siehe Ausbildungsdelegation I+D 2016a.

16 1987-1992 Vereinigung Schweizerischer Bibliothekare (VSB), 1992-2007 Verband der Bibliotheken und der Bibliothekare der Schweiz (BBS), 2008 mit der Schweizerischen Vereinigung für Dokumentation (SVD) fusioniert zu Bibliothek Information Schweiz (BIS). 


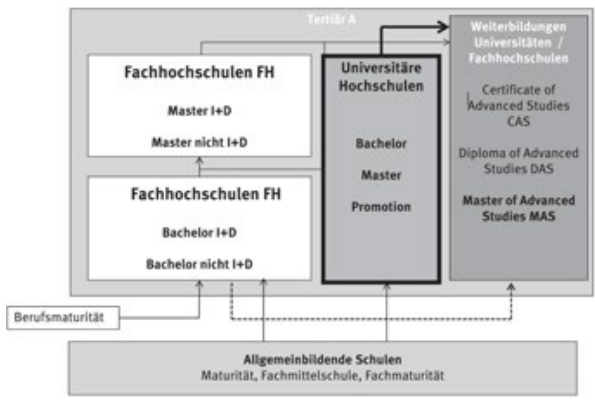

Abb. 2: Zugangswege zum Beruf „wissenschaftliche Bibliothekarin / wissenschaftlicher Bibliothekar" in der Schweiz. ${ }^{17}$

Aktuell gibt es in der Schweiz auf tertiärer Weiterbildungsstufe drei MAS-Studiengänge: den MAS ALIS (Archival, Library and Information Science) mit einem Schwerpunkt in Archivwissenschaften an den Universitäten Bern und Lausanne, den MAS BIW (Bibliotheks- und Informationswissenschaften) in Zürich und den MAS IS (Information Science) in Chur. Ein vierter, von 2013 bis 2015 im Kanton Tessin erstmals durchgeführter MAS in Library and Information Science ${ }^{18}$ wird momentan nicht angeboten. Derzeit nicht als MAS, sondern nur als Diploma of Advances Studies (DAS) durchgeführt wird der Weiterbildungsstudiengang in „Intelligence économique et veille stratégique" an der Haute École de Gestion in Genf.

In der Übersicht der aktuellen Schweizer Aus- und Weiterbildungsstudiengänge in Tabelle 1 erscheint der Begriff ,wissenschaftliche/r Bibliothekar/ in“ als Bezeichnung für den Vorläufer des MAS BIW, den ehemaligen „Kurs für Wissenschaftliche Bibliothekare (-innen)" des Verbandes der Bibliotheken und Bibliothekarinnen/Bibliothekare der Schweiz $(B B S)^{19}$. Dieser wurde von 1987 bis 2007 angeboten und bestand aus einer zweijährigen praktischen Ausbildung an einer wissenschaftlichen Bibliothek sowie einem berufsbegleitenden Theoriekurs an der Zentralbibliothek Zürich, wo der MAS BIW auch heute noch angesiedelt ist. Voraussetzungen für die Teilnahme waren ein universitärer ${ }^{20}$ Hochschulabschluss (Lizenziat/Diplom oder Promotion) und eine Praktikumsstelle oder eine bereits bestehende Anstellung an einer Universitätsbibliothek bzw. einer von der Aufsichtskommission anerkannten anderen Bibliothek. Der Kurs wurde bedarfsgerecht alle zwei Jahre durchgeführt mit jeweils ca. 10 zur Prüfung zugelassenen Inhaber und Inhaberinnen einer Praktikumsstelle sowie Gasthörerinnen und Gasthörer. Der vom Verband

17 Siehe Ausbildungsdelegation I+D 2016a mit Ergänzungen der Verf.

18 Vgl. SUPSI \& Sbt 2012/2013.

19 Heute BIS Bibliothek Information Schweiz.

20 Die Schweizer Fachhochschulen entstanden erst ab Mitte der 1990er Jahre. 
Tabelle 1: Bibliotheks- und informationswissenschaftliche MAS-Studiengänge (Ausbildungsdelegation I+D 2016b mit eigenen Hervorhebungen) ${ }^{21}$

Hochschulen - Weiterbildungen (CAS, DAS, MAS)

\begin{tabular}{|c|c|c|}
\hline Ausbildung & Zulassungsvoraussetzungen & Dauer \\
\hline $\begin{array}{l}\text { Archival. Library and Informa- } \\
\text { tion Science }\end{array}$ & $\begin{array}{l}\text { Master oder äquivalenter Ab- } \\
\text { schluss (Universität oder Fach- } \\
\text { hochschule) } \\
\text { + dreimonatige Praxiserfah- } \\
\text { rung in einer ABD-Institution } \\
\text { 'sur dossier* }\end{array}$ & $\begin{array}{l}\text { MAS: } 2 \text { Jahre, inkl. } 3 \text { Block- } \\
\text { seminare im In- und Ausland } \\
\text { (60 ECTS) } \\
\text { CAS: } 1 \text { Jahr (Gandstufe) ( } 20 \\
\text { ECTS) } \\
\text { berufsbegleitend }\end{array}$ \\
\hline $\begin{array}{l}\text { Bibliotheks- und Informa- } \\
\text { tionswissenschaften } \\
\text { (ehemals Wissen- } \\
\text { schaftliche/r Bibliothekar/ } \\
\text { in BBS) }\end{array}$ & $\begin{array}{l}\text { Hochschulabschluss auf der } \\
\text { Stufe eines Lizentiats oder } \\
\text { Masters } \\
\text { + Berufspraxis in einer Biblio- } \\
\text { thek oder l+D-Institution } \\
\text { Die Studiengangleitung ent- } \\
\text { scheidet über die Aufnahme } \\
\text { einer Kandidatin oder eines } \\
\text { Kandidaten }\end{array}$ & $\begin{array}{l}\text { MAS: } 4 \text { Semester (60 ECTS) } \\
\text { DAS: 3-4 Semester (34 ECTS) } \\
\text { CAS: 2-3 Semester (10 ECTS) }\end{array}$ \\
\hline
\end{tabular}

Fachhochschulen FH - Weiterbildungen (CAS, DAS, MAS)

\begin{tabular}{lll}
\hline $\begin{array}{l}\text { MAS Information Science - } \begin{array}{ll}\text { Mochschulabschluss (FH, Uni, } \\
\text { MAS IS }\end{array} \\
\text { ETH) oder vergleichbar } \\
\text { Ober die definitive Zulassung } \\
\text { entscheidet die Leitung des } \\
\text { MAS }\end{array}$ \\
\cline { 2 - 3 } $\begin{array}{l}\text { MAS Intelligence économique } \\
+ \text { Business intelligence MAS- }\end{array}$ & $\begin{array}{l}\text { Hochschulabschluss (FH, Uni, 2.5 Jahre (60 ETCS) } \\
\text { IEVS }\end{array}$ \\
$\begin{array}{l}\text { ETH) } \\
+ \text { mind. } 3 \text { Jahre Berufs- } \\
\text { erfahrung }\end{array}$ \\
\hline
\end{tabular}

21 Ausbildungsdelegation I+D (2016b). 


\begin{tabular}{|c|c|c|}
\hline Abschluss & Schulort/Bemerkungen & Kontaktadressen \\
\hline $\begin{array}{l}\text { Master of Advanced Studies in } \\
\text { Archival, Library and Information } \\
\text { Science, Universität Bern, } \\
\text { Université de Lausanne } \\
\text { Certificate of Advanced Studies in } \\
\text { Archival, Library and Information } \\
\text { Science, Universität Bern. } \\
\text { Université de Lausanne }\end{array}$ & $\begin{array}{l}\text { Universität Bern und } \\
\text { Lausanne } \\
\text { Unterrichtssprachen: } \\
\text { Deutsch, Französisch, } \\
\text { Englisch }\end{array}$ & $\begin{array}{l}\text { Universität Bern } \\
\text { Historisches Institut } \\
\text { Studienleitung Weiter- } \\
\text { bildungsprogramm in } \\
\text { Archiv-, Bibliotheks- und } \\
\text { Informationswissenschaft } \\
\text { Länggassstrasse } 493000 \\
\text { Bern } 9 \\
\text { www.archivwissenschaft. } \\
\text { ch }\end{array}$ \\
\hline $\begin{array}{l}\text { Master of Advanced Studies in } \\
\text { Bibliotheks- und Informationswis- } \\
\text { senschaften der Universität Zürich } \\
\text { Diploma of Advanced Studies in } \\
\text { Bibliotheks- und Informabonswis- } \\
\text { senschaften der Universität Zürich } \\
\text { Cerifícate of Advanced Studies } \\
\text { in Bibliotheks- und Informa- } \\
\text { tionswissenschaften der Universität } \\
\text { Zürich }\end{array}$ & $\begin{array}{l}\text { Zürich, Zentralbibliothek } \\
\text { Der Weiterbildungs- } \\
\text { studiengang wird von } \\
\text { der Universität Zürich } \\
\text { in Kooperation mit der } \\
\text { Zentralbibliothek Zürich } \\
\text { angeboten. }\end{array}$ & $\begin{array}{l}\text { Zentralbibliothek Zürich } \\
\text { Záhringerplatz } 68001 \\
\text { Zürich Fon +41 } 44268 \\
3130\end{array}$ \\
\hline
\end{tabular}

Master of Advanced Studies HTW Chur Siehe oben

FHO Information Science

Siehe oben

Master of Advanced Studies HEG Genève Siehe oben
HES-SO en Intelligence éco-
nomique et veille stratégique


ausgestellte Prüfungsausweis war im Unterschied zu den heutigen MAS-Studiengängen jedoch kein staatlich anerkannter Abschluss.

Der Ausbildungsgang für wissenschaftliche Bibliothekarinnen und Bibliothekare wurde 1987 erstmals durchgeführt. Die Professionalisierung erfolgte damit im Vergleich zu Deutschland, wo der (wissenschaftliche) „Berufsbibliothekar“ bereits 1893 geschaffen wurde, fast 100 Jahre später und auch deutlich später als bei den Schweizer Diplombibliothekarinnen und -bibliothekaren. ${ }^{22}$

Zusätzlich zum sogenannten „Zürcher Kurs“ für wissenschaftliche Bibliothekare und Bibliothekarinnen existierte bis 2009 in Genf das CESID (Certificat de spécialisation en information documentaire), das ebenfalls 1987 eingeführt worden war und zugleich als Kaderausbildung für Diplombibliothekarinnen und -bibliothekare diente. ${ }^{23}$ Vorläufer des Churer MAS IS war der Nachdiplomstudiengang (NDS) Information und Dokumentation.

Die heutigen MAS-Studiengänge haben unterschiedliche inhaltliche Schwerpunkte und unterscheiden sich auch bezüglich vorausgesetzter Vorbildung und Praxiserfahrung. Während die MAS-Studiengänge in Bern/Lausanne und Zürich einen Hochschulabschluss auf Masterstufe und einschlägige Berufspraxis im Rahmen von mindestens drei Monaten Vollzeit oder vier Monaten zu mindestens 50 Prozent verlangen, versteht sich der Churer MAS IS ${ }^{24}$ explizit als Ausbildungsgang für Absolventinnen und Absolventen eines Nicht-I+D-Studiums, die eine Zusatzqualifikation im Bereich Archiv, Bibliothek, Dokumentation oder Museum erwerben und in der Branche Fuß fassen wollen, und verlangt deshalb keine einschlägige praktische Erfahrung. $\mathrm{Zu}$ diesem Studiengang werden auch Inhaber und Inhaberinnen eines Bachelor-Abschlusses zugelassen. Der MAS ALIS ${ }^{25}$ hat spezifisch aktive oder zukünftige Führungskräfte von Archiven, Bibliotheken oder Informationseinrichtungen im Blick. Der MAS BIW ${ }^{26}$ richtet sich an Fachleute aus Bibliotheken und Informationseinrichtungen, die ihr Wissen im Bereich wissenschaftliche Bibliotheken im Hinblick auf Fach-, Führungs- und/oder Projektmanagementaufgaben vertiefen wollen.

Nicht mehr vorausgesetzt für den Abschluss eines bibliothekarischen MASStudiengangs wird heute eine Praktikumsstelle oder ein Volontariat. Einige große Schweizer Bibliotheken wie die Universitätsbibliotheken Basel und Bern, die Zentralbibliothek Zürich oder die Schweizerische Nationalbibliothek bieten

22 Vgl. Barth \& Schneider 1997, S. 90-91; Siebert 2014, S. 27.

23 Vgl. Barth \& Schneider 1997, S. 91-92.

$24 \mathrm{http} / /$ www.htwchur.ch/informationswissenschaft/weiterbildung/mas-in-information-science.html (27.07.2016).

25 http://www.archivwissenschaft.ch/ (27.07.2016).

26 http://www.mas-biw.uzh.ch (27.07.2016). 
jedoch weiterhin im Zweijahresrhythmus wissenschaftliche Ausbildungsstellen für je eine bis zwei Personen an und fokussieren dabei auf Kandidaten und Kandidatinnen mit einem universitären Masterabschluss. ${ }^{27}$ Das Interesse an diesen wenigen wissenschaftlichen Praktikums-/Volontariatsstellen ist mit bis $\mathrm{zu} 70$ oder mehr Bewerbungen pro Bibliothek und Ausbildungszyklus sehr hoch.

Einige große Bibliotheken bieten keine eigenen wissenschaftlichen Ausbildungsstellen (mehr) an, sondern entsenden bereits angestellte Mitarbeitende bei Bedarf in einen bibliothekarischen Weiterbildungsstudiengang in der Schweiz oder zunehmend auch im Ausland, insbesondere nach Deutschland. Dies ist nur eines von verschiedenen Indizien für eine zunehmende Diversifizierung und Durchlässigkeit des Berufsfeldes und der Zugangswege dazu.

Mittlerweile führen unterschiedlichste (Ausbildungs-)Wege zum Beruf „wissenschaftliche Bibliothekarin/wissenschaftlicher Bibliothekar“ im Sinne der oben abgeleiteten breiten Definition, sei es auf der klassischen Route via nichtinformationswissenschaftlichen Masterabschluss und postgradualen bibliothekswissenschaftlichen MAS-Studiengang, sei es via I+D-Ausbildung bis hin zum konsekutiven Masterabschluss oder sei es via Quereinstieg als Akademiker oder Akademikerin ohne bibliothekarischen Hintergrund. Aus der Perspektive von Fachhochschulbibliotheken als noch wachsender Sektor im wissenschaftlichen Bibliothekswesen wären mehr Absolventinnen und Absolventen von nicht-universitären Masterstudiengängen wie Design, Ingenieurwesen etc. mit einer bibliothekarischen Zusatzausbildung erwünscht, um die entsprechenden Fachbereiche an diesen Einrichtungen fachkompetent zu betreuen. Universitätsbibliotheken fördern ihren Nachwuchs weiterhin via wissenschaftliche Volontariatsstellen - die im Unterschied zu früher bezahlt werden ${ }^{28}$ - stellen aber auch Mitarbeitende mit akademischem Hintergrund ohne bibliothekarische Zusatzausbildung an, teilweise mit der Auflage, nach der Anstellung einen bibliothekarischen MAS-Studiengang zu absolvieren.

27 Zentralbibliothek Zürich (http://www.zb.uzh.ch/ausbildung/mas/zusatzinfos/003697/index. html.de); Universitätsbibliothek Basel (http://www.ub.unibas.ch/ub-hauptbibliothek/wir-ueberuns/weiteres/offene-stellen-ausbildungsplaetze/), Schweizerische Nationalbibliothek (http:// www.nb.admin.ch/org/00863/00869/00884/index.html?lang=de); Universitätsbibliothek Bern (http://www.unibe.ch/universitaet/dienstleistungen/universitaetsbibliothek/ueber_uns/ausbildungsangebote/mas_studiengang_bibliotheks_und_informationswissenschaften/index_ger. html) (jeweils 27.07.2016). In Bern wird das wissenschaftliche Praktikum explizit als Ausbildung zur wissenschaftlichen Bibliothekarin bzw. zum wissenschaftlichen Bibliothekar bezeichnet. Vgl. dazu auch Ingold 2015.

28 Vgl. Barth \& Schneider 1997, S. 80. Von der ersten wissenschaftlichen Bibliothekarin in Basel, die 1934/35 ihr Volontariat wie damals üblich „ohne Vergütung“ absolvierte, berichten Oeggerli \& Steinegger 1995, S. 204. 


\section{Die Diskussion um das Berufsbild}

In der Schweiz ist „Wissenschaftliche Bibliothekarin / Wissenschaftlicher Bibliothekar" weder ein offizieller Ausbildungsberuf noch eine geschützte Berufsbezeichnung und war dies auch nie. Zwar bestand in den 1930er Jahren auch in der Schweiz eine Dreiteilung in „oberen“ (Deutschland = höheren), „mittleren“ (= gehobenen) und ,unteren“ (= mittleren) Dienst, und formal waren nur die - damals noch existierenden - wissenschaftlichen Beamten zur Führung des Titels „Bibliothekar“ berechtigt. De facto jedoch stellten schon früh Frauen den Großteil des diplomierten Personals. Sie waren während Jahrzehnten die dominierende Berufsgruppe in der Schweizer Bibliothekslandschaft, für die auch der Begriff „Bibliothekar/Bibliothekarin“ im allgemeinen Sprachgebrauch stand.

Gemäß Barth \& Schneider ${ }^{29}$ lebten wissenschaftliche und Diplombibliothekare weitgehend harmonisch zusammen. Senser stellt 1991 fest: „Der höhere Dienst übernimmt nach einem abgeschlossenen Hochschulstudium überwiegend - aber nicht nur - Aufgaben im Bereich des Fachreferats und leitende Funktionen. Alle übrigen bibliothekarischen Tätigkeiten werden von dem mittleren Dienst geleistet. Eine weitere Unterteilung in gehobenen und mittleren Dienst wie in der Bundesrepublik Deutschland gibt es nicht. “" ${ }^{30}$

Andererseits hatte der Bibliothekarsberuf allgemein und derjenige der wissenschaftlichen Bibliothekare und Bibliothekarinnen im Besonderen keinen sehr hohen Status. Barth \& Schneider diagnostizieren ein „Leiden am Berufsimage, das sich durch das ganze Jahrhundert zieht ${ }^{\text {“31 }}$. Arbeit in einer Bibliothek hatte offenbar nur geringes Prestige und wurde für Akademiker als unwürdig betrachtet. Professionelle bibliothekarische Zusatzqualifikationen zum akademischen Abschluss wurden von den Anstellungsinstanzen als verzichtbar erachtet. Senser stellte fest: „Die von Marcel Godet ${ }^{32}$ geforderte Prüfungsordnung für den höheren Dienst steht immer noch aus. Es liegt bisher weder ein Berufsbild im Sinne einer zusammenfassenden Beschreibung aller zu übernehmender Funktionen noch eine geregelte Ausbildungsordnung vor. Hermann Eschers ${ }^{33}$ Schilderung, wie er sich Ende des 19. Jahrhunderts sein bibliothekarisches Wissen aneignete, kann auch heute noch so im Lebenslauf eines Bibliothekars stehen, durch Lernen

29 Barth \& Schneider 1997, S. 110.

30 Senser 1991, S. 144.

31 Barth \& Schneider 1997, S. 111-112.

32 Marcel Godet, 1877-1949, Direktor der Schweizerischen Landesbibliothek und IFLA-Präsident (Kurzbiografie in Barth \& Schneider 1997, S. 153-155).

33 Hermann Escher, 1858-1938, Direktor der Zentralbibliothek Zürich (Kurzbiografie in Barth \& Schneider 1997, S. 151-153). 
von älteren Kollegen, durch autodidaktisches Fachliteraturstudium, durch Auslandsreisen." ${ }^{\text {34 }}$

Von 200 Jahren Berufsbild-Debatte ${ }^{35}$ sind die wissenschaftlichen Bibliothekarinnen und Bibliothekare in der Schweiz weit entfernt. Berufsbild-Diskussionen wie in Deutschland, die vor allem das berufliche Selbstverständnis und die Gewichtung von eigener wissenschaftlicher Tätigkeit gegenüber Verwaltungsaufgaben betreffen, gab und gibt es in dieser Form nicht. Das hängt unter anderem damit zusammen, dass weder ein Beamtenstatus noch eine ausgeprägte hierarchische Differenzierung von bibliothekarischen Tätigkeitsfeldern bestehen. „Polarisierende Leitbilder“ und anstellungstechnische Fragen wie „Laufbahnbefähigungen, Qualifikationsebenen und Eingruppierungen“36 fallen damit als Streitpunkte weitgehend weg.

In deutschen Berufsbildern lassen sich in der Regel drei Tätigkeitsbereiche differenzieren: Fachreferat (Auswahl und Erschließung, Benutzerberatung und Informationskompetenz), Leitungs- und Managementaufgaben sowie Entwicklung neuer Dienstleistungen und Konzepte mit entsprechender Projektarbeit. Vor allem vor dem Hintergrund neuer Managementaufgaben und neuer Aufgaben im Internetzeitalter entzündete sich in Deutschland Ende der 1990er Jahre eine heftige und teils polemisch geprägte Debatte. ${ }^{37}$ Auf der einen Seite stand die Betonung des wissenschaftlichen Bibliothekars „als Fachreferent und Informationsspezialist“38 - mit einer durchaus aktiven Rolle als Informationsspezialist, wie sie auch heute, immerhin fast 20 Jahre später, im Konzept des Embedded Librarian im zeitgenössischen Gewand diskutiert wird. Demgegenüber betonten andere Autoren die Managementaufgaben, das „vielfältige Tätigkeitsspektrum der Bibliotheksverwaltung “39.

Versteht man unter der Erweiterung des „klassischen“ Fachreferats in Richtung Embedded Librarian eine Erweiterung um moderne wissenschaftliche bzw. wissenschaftsnahe Dienstleistungen und berücksichtigt, dass im Bereich von Leitung und Management ebenfalls Projektarbeiten angesiedelt sind, bricht die starre Dichotomie auf: Fachreferat und Leitungsaufgaben fordern und fördern die Entwicklung neuer bibliothekarischer Services und Produkte. Aktuell entstehende Arbeitsbereiche zu Bibliometrie oder zum Forschungsdatenmanagement lassen sich durchaus als konsquente Weiterentwicklung bibliothekarischer

34 Senser 1991, S. 146.

35 Vgl. Siebert \& Lemanski 2014.

36 Plappert 2015.

37 Vgl. u. a. Bosserhoff 2008 und Jochum 2014.

38 Oehling 1998, S. 248.

39 Te Boekhorst et al. 1998, S. 690. 
Dienste durch die technologische und wissenschaftliche Entwicklung begreifen, nicht unbedingt als neue Berufszweige oder -wege.

Trotzdem war und ist der bibliothekarische Berufsstand auch in der Schweiz Thema von Diskussionen, die hauptsächlich mit den Veränderungen der Ausbildungslandschaft der 1990er Jahre zusammenhängen. Damals erfolgte eine stärkere Differenzierung und Profilierung der bibliothekarischen Berufsgruppen, und die 1988 bzw. 1992 neu gegründeten Interessengruppen der DiplombibliothekarInnen ${ }^{40}$ und der wissenschaftlichen BibliothekarInnen ${ }^{41}$ beschäftigten sich verstärkt mit Ausbildungsfragen und Berufsbildern.

\section{Das Berufsbild und die Berufsverbände}

In seinem Vierjahresplan 1993 bis 1996 stellte der nationale Berufsverband BBS „die Ausarbeitung von neuen, aufeinander abgestimmten Berufsbildern für die bibliotheksspezifischen Berufe“ sowie „ein Gesamtkonzept der bibliothekarischen Ausbildung“ als Zielvorgabe auf. ${ }^{42}$ Das geplante Rahmenprogramm für die Ausbildung „Bibliothekar, Dokumentalist, Archivar (BDA)“ sollte die Durchlässigkeit zwischen den drei verwandten Tätigkeitsfeldern gewährleisten. Zudem sollte eine ,gemeinsame, koordinierte und integrierte Fach- und Kaderausbildung“ auf Nachdiplomstufe „unnötige Konkurrenzsituationen“ innerhalb der Ausbildungsstufen und Berufsgruppen entschärfen, insbesondere zwischen „Diplompersonal und wissenschaftlichem Personal“43. Für das Nachdiplomstudium sollte auch nicht zwischen Fachhochschul- und Universitätsabsolventen/-absolventinnen unterschieden werden.

Unabhängig davon erstellte die BBS-Aufsichtskommission des 1987 eingeführten Zürcher Kurses für wissenschaftliche Bibliothekare bereits 1993 ein kurzes Berufsbild „Wissenschaftlicher Bibliothekar“ als Grundlage für diesen Ausbildungsgang. ${ }^{44}$ Darin wurden Aufgaben und Tätigkeiten in folgende vier Bereiche gegliedert:

- Organisatorische Aufgaben, unterteilt in klassische Leitungsaufgaben und Datenverarbeitung

40 Interessengruppe Schweizer Diplombibliothekare/innen SBD, 1988-2008.

41 Von 1992 bis 2013 unter der Bezeichnung „Interessengruppe der Wissenschaftlichen Bibliothekarinnen und Bibliothekare der Schweiz“, seither „Interessengruppe Wissenschaftliche BibliothekarInnen Schweiz“, mit gleichbleibender Kurzform „IG WBS“.

42 Vgl. IG WBS 1993a.

43 Vgl. IG WBS 1994, S. 2-3.

44 Vgl. IG WBS 1993b, S. 1 und Anhang. 
- Wissenschaftliche Fachaufgaben

- Aufgaben in der Lehre sowohl für den Berufsnachwuchs wie für Bibliotheksbenutzer

- Besondere Aufgaben wie Projekte, Stabsfunktionen etc.

Vorausgesetzt wurden ein abgeschlossenes Fachstudium einer wissenschaftlichen Hochschule, eine breite Allgemeinbildung sowie PC- und EDV-Kenntnisse.

In diesen Text eingeflossen war zumindest teilweise der Input der IG WBS. Der Vorstand IG WBS hielt in den Mitteilungen an seine Mitglieder fest: „Wegen der vorgegebenen engen Grenzen mussten diverse wichtige Aspekte übergangen werden, die die IG in einem eigenen Papier zum Thema Berufsbild aufarbeiten will (Kursziele, Berufsmöglichkeiten....)“45. Zu diesem Zweck wurde eine interne Arbeitsgruppe „Berufsbild“ (später „Ausbildung und Berufsbild“) eingesetzt.

Im Jahr 1997 erschien die von der IG WBS verfasste und vom BBS herausgegebene zehnseitige Berufsbild-Broschüre „Wissenschaftliche Bibliothekarin / Wissenschaftlicher Bibliothekar“ (vgl. Abb. 3) ${ }^{46}$ mit folgendem Inhalt:

- Tätigkeitsbereiche:

- Informationsmanagement in einer wissenschaftlichen Bibliothek, einer Spezialabteilung, einem Archiv oder einer Dokumentationsstelle

- Kaderaufgaben in einer wissenschaftlichen Bibliothek oder einem Archiv

- Selbständige Tätigkeit (Information Brokerage, Beratung).

- Berufspraxis:

- Informationsvermittlung, bereitstellung, erschließung und erhaltung

- Kaderaufgaben

- Ausbildungsgänge: BBS-Kurs für Wissenschaftliche Bibliothekare (-innen), CESID, Nachdiplomstudium Information und Dokumentation.

45 IG WBS 1992, S. 2.

46 Vgl. BBS 1997. 


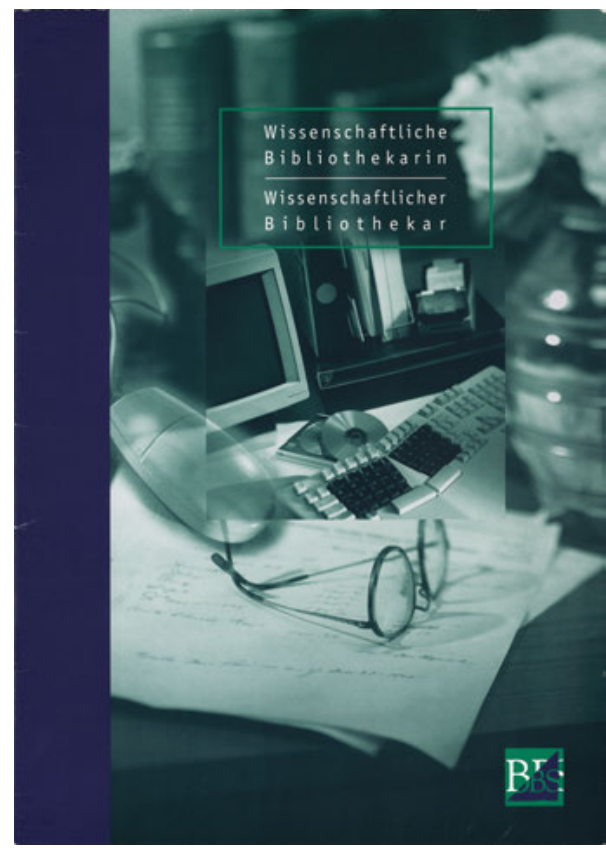

Abb. 3: Erste Schweizer Berufsbildbroschüre „Wissenschaftliche Bibliothekarin / Wissenschaftlicher Bibliothekar" (BBS 1997). ${ }^{47}$

2012 bis 2013 wurde das Berufsbild „Wissenschaftliche Bibliothekarin / Wissenschaftlicher Bibliothekar" von einer internen Arbeitsgruppe der IG WBS grundlegend überarbeitet und neu gestaltet (vgl. Abb. 4), da sich die Ausbildungslandschaft in den Jahren zuvor stark verändert hatte und der Text auch inhaltlich teilweise veraltet war. Die Ausgangsfrage für die Überarbeitung lautete: „Wer sind und was tun wissenschaftliche Bibliothekarinnen und Bibliothekare in der Berufspraxis?“ Dazu hatte 2011 ein World-Café mit Mitgliedern der IG WBS stattgefunden ${ }^{48}$, das als Grundlage für die Arbeitsgruppe diente.

Nach dem Motto „Form follows Function“49 oder vielmehr „Profession follows Practice“ basiert das Berufsbild 2013 auf dem realen Tätigkeitsspektrum von Mitgliedern der IG WBS und anderen Personen mit akademischer Vorbildung in Schweizer Bibliotheken. Es unterscheidet die vier Tätigkeitsfelder

- Wissenschaft und Forschung

- Management und Leitung

- Technologie und Innovation

47 Vgl. BBS 1997.

48 Vgl. Bellanger \& Ingold 2011.

49 Haubfleisch 2014, S. 132. 
- Projekte und Spezialaufgaben

und enthält zu jedem dieser Tätigkeitsfelder eines oder mehrere Porträts. ${ }^{50}$

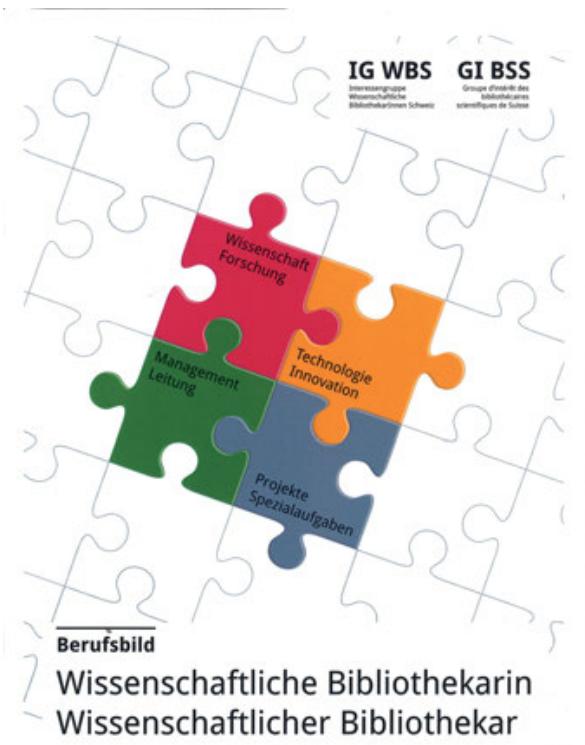

Das neue Schweizer Berufsbild stieß in in- und ausländischen Fachkreisen auf positive Resonanz, weckte dank erfolgreicher Kommunikation unter anderem das Interesse von Berufsberatungsstellen und vermochte es, den Bekanntheitsgrad des Berufes in einer breiteren Öffentlichkeit zu steigern. ${ }^{52}$

Inhaltlich bildet das neue Berufsbild die auch von Bonte skizzierte zunehmende Diversifizierung und Spezialisierung des „Wissenschaftlichen Dienstes in Bibliotheken“ im Sinne von ,akademisch vorgebildeten Beschäftigten“ bereits zu weiten Teilen ab. ${ }^{53}$ Damit bricht es nicht etwa mit seinen Vorgängerversionen, sondern greift die bereits in diesen angelegte Vielfalt des Berufsfeldes auf und passt sie neueren Entwicklungen an. Am wenigsten prominent vertreten ist die von Bonte erwähnte Kategorie „ForschungsbibliothekarInnen“, die die „Infrastruktur- und Themenentwicklung einer Disziplin oder Teildisziplin spürbar

50 Vgl. IG WBS 2013.

51 IG WBS 2013.

52 Vgl. Schaub 2014a und 2014b; Schaub \& Gavillet 2014.

53 Bonte 2014. 
unterstützen und für die Bibliothek regelmäßig Drittmittel akquirieren können“"54. Das hängt wohl nicht zuletzt mit den anderen Vergabepraktiken der Schweizer Forschungsförderinstitutionen wie dem Nationalfonds zusammen, die im Unterschied beispielsweise zur DFG keine Bibliotheksprojekte finanzieren. Mit dem nationalen Programm „Wissenschaftliche Information: Zugang, Verarbeitung und Speicherung“ steht aber zumindest in den nächsten Jahren eine Finanzierungsmöglichkeit für hochschulübergreifende Forschungsprojekte unter Beteiligung von Bibliotheken zur Verfügung.

Bezüglich eigener wissenschaftlicher Tätigkeit von wissenschaftlichen Bibliothekarinnen und Bibliothekaren herrscht in der Schweiz eine pragmatische Einstellung. Ein Konflikt zwischen Wissenschaft oder Verwaltung als bestimmenden Tätigkeitsfeldern wie in Deutschland ${ }^{55}$ existiert in dieser Form nicht. Zwar gibt es durchaus gelegentlich Klagen über fehlende Anerkennung „innerhalb des Sozialsystems Universität“56, doch grundsätzlich gilt auch heute noch: „Der Bibliothekar dient der Wissenschaft, macht aber keine aus seiner Tätigkeit“ ${ }^{* 57}$ - was nicht bedeutet, dass wissenschaftliche Betätigung nicht stattfindet oder erwünscht ist.

Die Gleichsetzung von wissenschaftlichen Bibliothekarinnen und Bibliothekaren mit dem Tätigkeitsfeld Fachreferat existiert auch in der Schweiz, war und ist aber weniger stark ausgeprägt als in Deutschland ${ }^{58}$, da schon immer andere Aufgabenfelder zum Berufsprofil gehört haben. ${ }^{59}$ Zudem ist eine deutliche Weiterentwicklung hin zum „Dienstleistungsbibliothekar“60 spürbar. Im Berufsbild 2013 erscheinen „Dienstleistung“ oder davon abgeleitete Begriffe insgesamt 15 Mal auf 23 Seiten; das ist im Vergleich zum Berufsbild 1997 mit drei Mal auf zehn Seiten doppelt so häufig. Ein dienstleistungsorientiertes Profil, eine „konsequente, berufsalltäglich auf ihre Aktualität und Modernität überprüfte Hinwendung zum Kunden“61 als Grundlage für eine positive Fortentwicklung, Dynamik und Flexibilität des Berufes ist also zumindest auf der normativen Ebene deutlich spürbar.

In Bezug auf eine (zusätzliche) bibliothekarische Ausbildung bleibt das neue Schweizer Berufsbild flexibel und bildet damit die bestehende Praxis ab. Es deklariert die Ausbildung aber klar als Desiderat, indem es wissenschaftliche

54 Bonte 2014, Folie 18.

55 Vgl. z. B. Bosserhoff 2008 und Jochum 2014.

56 Enderle 2014, S. 56.

57 IG WBS 1993b.

58 Vgl. z.B. Ball 2011 und Bonte 2011.

59 Vgl. IG WBS 1993b, BBS 1997 und IG WBS 2013.

60 Siebert 2014, S. 33.

61 Barth \& Brugbauer 2014, S. 97. 
Bibliothekarinnen und Bibliothekare als Mitarbeitende definiert, „die über ein abgeschlossenes Hochschulstudium und vorzugsweise eine bibliothekarische Zusatzausbildung verfügen“62.

Eher zurückhaltend bleibt das Schweizer Berufsbild 2013 in Bezug auf den Bereich Informatik und Informationstechnologie. Diese wird in erster Linie als äußerer, das Berufsfeld prägender Einfluss und als Weiterbildungsoption beschrieben, aber nicht als grundlegendes Element der Ausbildungsgänge für wissenschaftliches Bibliothekspersonal gefordert, in denen es noch (zu) wenig Berücksichtigung findet. Hier besteht die Gefahr einer wachsenden Diskrepanz zwischen der steigenden Nachfrage aus der Bibliothekspraxis und dem knappen Angebot an bibliothekarisch ausgebildetem wissenschaftlichen Personal mit entsprechenden Fähigkeiten.

Als „wissenschaftliche Bibliothekarinnen“ und „wissenschaftliche Bibliothekare“ verstehen sich in der Schweiz wohl am ehesten die Absolventen bzw. Absolventinnen eines wissenschaftlichen Volontariats oder Praktikums mit begleitendem bibliothekarischem Weiterbildungsstudiengang. Bei den Teilnehmenden von MAS-Studiengängen und Quereinsteigenden ist das vermutlich weniger der Fall. Auch die Arbeitgeberinstitutionen legen eher wenig Wert auf die entsprechende Berufsbezeichnung. Die Stellenausschreibungen der letzten Jahre in der deutschsprachigen Schweiz zeigen eine große Vielfalt nicht nur bezüglich der zu besetzenden Aufgabenbereiche, sondern auch bezüglich der verwendeten Berufsbezeichnungen und verlangten Voraussetzungen. ${ }^{63}$ Diese Vielfalt entspricht jedoch der Realität auf dem Arbeitsmarkt und wird künftig wohl noch zunehmen.

Im Vergleich zur beruflichen Grundbildung, die zum staatlich anerkannten Abschluss „Fachfrau/Fachmann Information und Dokumentation EFZ“64 führt und im Web und in Fachpublikationen detailliert beschrieben und dokumentiert ist $^{65}$, hat „wissenschaftliche Bibliothekarin“ oder „wissenschaftlicher Bibliothekar“ als Weiterbildungsberuf eine deutlich geringere Präsenz. Immerhin stößt aber heute direkt auf das Schweizer Berufsbild der IG WBS, wer „wissenschaftliche Bibliothekarin“ oder „wissenschaftlicher Bibliothekar“ in eine Suchmaschine eingibt.

Nun kann es selbst auferlegte Aufgabe der einschlägigen Interessengruppe bzw. des nationalen Branchenverbandes sein, die Berufsbezeichnung allgemein

62 IG WBS 2013, S. 19, Hervorhebung der Verf.

63 Vgl. Schaub 2014b und Ingold 2015.

64 EFZ steht für Eidgenössisches Fähigkeitszeugnis. Von 1998 bis 2011 lautete die Berufsbezeichnung „Informations- und Dokumentationsassistent/in“.

65 Vgl. Ingold 2015. 
bekannter zu machen, Arbeitgeberinstitutionen bezüglich klarer Anforderungen und Terminologie in Stellenausschreibungen zu sensibilisieren und das Selbstverständnis der Berufsangehörigen zu fördern. Für Absolventinnen und Absolventen der bibliothekswissenschaftlichen Studiengänge ist es aber existenziell wichtig, dass ihre erworbenen Qualifikationen den Anforderungen des Arbeitsmarktes entsprechen, und Arbeitgeber müssen grundsätzlich die Möglichkeit haben, für ihre freien Stellen die passenden Persönlichkeiten unabhängig von formaler Qualifikation und Berufsbezeichnung zu gewinnen. ${ }^{66}$

Das aktuelle Schweizer Berufsbild dient deshalb bewusst vor allem der Orientierung und Information von potenziellen Berufseinsteigerinnen und -einsteigern, indem es die Vielfalt der aktuellen Berufspraxis abbildet. Es bewirbt damit unter anderem aktiv die bibliothekswissenschaftlichen Weiterbildungsstudiengänge, ohne allerdings einen direkten Einfluss auf deren Inhalte zu haben und ohne den Absolventinnen und Absolventen eine eindeutige Berufsidentität anbieten zu können oder zu wollen.

Pragmatisch entwickelte auch der Verein Deutscher Bibliothekarinnen und Bibliothekare (VDB) 2013/14 ein Positionspapier zur Qualifikation als Wissenschaftliche Bibliothekarin / Wissenschaftlicher Bibliothekar und ging dabei von derzeitigen Tätigkeitsfeldern aus:

Gemeinsam sind allen Tätigkeitsgebieten speziell der wissenschaftlichen Bibliothekarinnen und Bibliothekare jedoch das konzeptionelle und strategische Arbeiten in einem durch starke Veränderungen geprägten Umfeld (...). So sehr sich durch bildungspolitischen und informationstechnologischen Fortschritt, Veränderungen am Medienmarkt oder durch ein verändertes Zusammenarbeiten aller bibliothekarischen Beschäftigten die Tätigkeiten und Aufgaben (...) im Detail weiterentwickeln, lassen sich doch einige klassische Einsatzfelder nach wie vor differenzieren. ${ }^{67}$

Dieses VDB-Positionspapier folgt den eher klassischen Arbeitsfeldern von Fachreferatsarbeit, Projekt- und Managementaufgaben und betont innerhalb dieser Schwerpunkte moderne Aufgaben und Herausforderungen. Zum Beispiel wird durch Approval Plans Erwerbung nicht obsolet, sondern die Tätigkeit verändert sich innerhalb des Schwerpunktes Erwerbung. Auch Dienstleistungen für wissenschaftliche Fachkulturen zu erbringen, ist an sich kein neues Aufgabengebiet. Durch Open Access, Forschungsdatenmanagement u. a. entstehen aber neue Ausprägungen und Anforderungen. Aus diesen Tätigkeiten werden Kernkompetenzen abgeleitet und hieraus wiederum Anforderungen benannt, die für Aus- und Weiterbildung bedeutsam sind: „Ein veränderter Technologie-und Medienmarkt

66 Vgl. Haubfleisch 2014, S. 133.

67 VDB 2014, S. 2. 
führt zu veränderten innerbetrieblichen Schwerpunkten und Arbeitsteilungen zwischen den bibliothekarischen Qualifikationsebenen. (...) Die Entwicklung der Informationstechnologie, neu entstehende Tätigkeitsschwerpunkte und die mit dem Berufsfeld verbundenen Leitungsaufgaben erfordern sowohl fachliche und überfachliche Qualifikationen eines Hochschulstudiums als auch eine postgraduale infrastrukturelle Ausbildung“68. Wie diese beidseitige Qualifizierung konkret gestaltet werden kann - das bleibt offen und muss es angesichts der veränderten Ausbildungswege und sogenannten Quereinsteigern in den Beruf wohl auch bleiben.

\section{Ausblick}

Die Diskussion um ein berufliches Selbstverständnis scheint keineswegs abgeschlossen zu sein. ${ }^{69}$ Das Bedürfnis nach einer Standortbestimmung im Spannungsfeld zwischen individuellem Selbstverständnis und konkreten Tätigkeiten, aber von Tradition und Außenwahrnehmung des Berufes (auch unter Laufbahnund Vergütungsaspekten) scheint in diesem dynamischen Berufsfeld in gewissen Zyklen virulent zu werden.

Selbst die Berufsbezeichnung an sich steht zur Debatte: Noch lässt sich nicht abschätzen, ob und mit welchen Inhalten versehen die Bezeichnung „Wissenschaftliche Bibliothekarin / Wissenschaftlicher Bibliothekar“ Bestand haben wird, oder ob man zukünftig von „Wissenschaftliche Bibliotheksmitarbeiterin, Wissenschaftlicher Bibliotheksmitarbeiter“, „Wissenschafts-BibliothekarIn“ oder gar „Experte/Expertin für Wissenschaftsdienstleistungen“ sprechen wird.

Wie auch immer der sich mit großer Dynamik ändernde Beruf in Zukunft heißen mag, entscheidend bleiben die von seinen Angehörigen erbrachten relevanten Dienstleistung für die Zielgruppen in der Wissenschaft und ein wertvoller Beitrag zur Zukunft der Informationsgesellschaft!

68 VDB 2014, S. 7.

69 Der VDB etwa hat das Positionspapier bim Bibliothekartag in Bremen 2014 vorgestellt, der Bayerische Landesverband hat im September 2014 eine Fortbildungsveranstaltung zum Berufsbild veranstaltet; im Rahmen eines Round Table wurde das Positionspapier im März 2015 diskutiert. O-bib - das offene Bibliotheksjournal widmete mit 3/2015 dem Berufsbild einen Themenschwerpunkt und veröffentlichte hier vier Beiträge der VDB-Fortbildung vom September 2014. https://www.o-bib.de/issue/viewIssue/2015H3/17 (29.6.2016). 


\section{Literatur}

\section{Zitierte Literatur und Internetquellen}

Ausbildungsdelegation I+D (2016a). Bildungsschema. http://www.ausbildung-id.ch/fileadmin/ redaktion/Dokumente/Home/01_Bildung/03_Tertiaere_Bildung_Weiterbildung/ Bildungsschema.pdf (21.04.2016).

Ausbildungsdelegation I+D (2016b). Überblick Aus- und Weiterbildungen. http://www. ausbildung-id.ch/fileadmin/redaktion/Dokumente/Home/01_Bildung/03_Tertiaere_ Bildung_Weiterbildung/Aus-_und_Weiterbildungen.pdf (21.4.2016).

Ball, R. (2011). Das Ende aller Nischen: Der künftige Platz des Wissenschaftlichen Bibliothekars im modernen Dienstleistungsbetrieb. Vortrag, gehalten auf dem 100. Deutschen Bibliothekartag vom 7.-10. Juni 2011 in Berlin. urn:nbn:de:0290-opus-10098

Barth, D. \& Brugbauer, R. (2014). Zwischen Fachreferat, Management und Informationstechnologie: Zur Berufswirklichkeit des wissenschaftlichen Dienstes in universitären Bibliothekssystemen. In I. Siebert \& T. Lemanski (Hrsg.), Bibliothekare zwischen Verwaltung und Wissenschaft: 200 Jahre Berufsbilddebatte (S. 81-97). (Zeitschrift für Bibliothekswesen und Bibliographie, Sonderband 111). Frankfurt am Main: Klostermann.

Barth, R. \& Schneider, G. (1997). Bibliotheken, Bibliothekarinnen und Bibliothekare in der Schweiz. Hundert Jahre bibliothekarischer Branchenverband 1897-1997. Vevey: Editions de l'Aire.

BBS - Verband der Bibliotheken und der Bibliothekarinnen/Bibliothekare der Schweiz BBS (Hrsg.) (1997). Wissenschaftliche Bibliothekarin / Wissenschaftlicher Bibliothekar. Bern: BBS. http://www.igwbs.ch/wp-content/uploads/Berufsbild-1997.pdf (17.04.2016).

Bellanger, S. \& Ingold, M. (2011). World Café zum Berufsfeld „Wissenschaftliche/r Bibliothekar/ in": Ein Rückblick auf die Veranstaltung vom 31. Mai 2011 in Bern. IG WBS, Rundbrief an die Mitglieder (55), 3-4. http://www.igwbs.ch/wp-content/uploads/2013/01/Rundbrief_55. pdf (28.04.2016).

Berufsberatung.ch (2016). Berufe und Ausbildungen. Beruf: Wissenschaftliche/r Bibliothekar/ in. http://www.berufsberatung.ch/dyn/1203.aspx?id=6113\&id_zihlmann=20 (17.04.2016).

BIB - Berufsverband Information Bibliothek e.V.: Kommission Ausbildung und Berufsbilder (2016): Berufsbild Wissenschaftliche/r Bibliothekar/in. http://www.bib-info.de/ kommissionen/kommission-ausbildung-und-berufsbilder/ausbildung/studium-masterreferendariat/berufsbild-wiss-bibliothekarin.html (02.05.2016).

Bonte, A. (2014). Der wissenschaftliche Dienst in der Digitalen Bibliothek: Was kommt nach dem Fachreferentensystem? Vortrag zur Veranstaltung „Berufsbild Wissenschaftliche( $r$ ) Bibliothekar(in) heute“ vom 30.9.2014 in Frankfurt/Main http://de.slideshare.net/ Achim_Bonte/der-wissenschaftliche-dienst-in-der-digitalen-bibliothek-was-kommt-nachdem-fachreferentensystem-39650127 (28.4.2016).

Bonte, A. (2011). Theoretisches Berufsbild und berufliche Praxis: Der höhere Dienst in der Digitalen Bibliothek. Vortrag, gehalten auf dem 100. Deutschen Bibliothekartag in Berlin, 8.6.2011. http://de.slideshare.net/Achim_Bonte/theoretisches-berufsbild-und-beruflichepraxis-der-hhere-dienst-in-der-digitalen-bibliothek (27.07.2016).

Bosserhoff, B. (2008). Wissenschaftlicher Bibliothekar - Berufsstand in der Legitimationskrise? Ein Rückblick auf die Debatte von 1998. Bibliotheksdienst 42(11), 1161-1171. 
Braun, K. \& Brunenberg-Piel, U. (2014). Fachreferat heute. Analyse des Berufsbildes von Fachreferenten anhand von Stellenanzeigen der Jahre 2003 bis 2013. In A. Oßwald et al. (Hrsg.). MALIS-Praxisprojekte 2014: Projektberichte aus dem berufsbegleitenden Masterstudiengang Bibliotheks- und Informationswissenschaft der Fachhochschule Köln (S. 189-210). (b.i.t.online - Innovativ, Bd. 50). Wiesbaden: Dinges \& Frick.

Brockhaus Enzyklopädie Online (2015). „Berufsbild“. Version vom 1.1.2012.https://fh-nordws. brockhaus-wissensservice.com/brockhaus/berufsbild (8.5.2015).

Enderle, W. (2014). Selbstverantwortliche Pflege bibliothekarischer Bestände und Sammlungen: Zur Genese und Funktion wissenschaftlicher Fachreferate in Deutschland 1909-2011. In I. Siebert \& T. Lemanski (Hrsg.), Bibliothekare zwischen Verwaltung und Wissenschaft: 200 Jahre Berufsbilddebatte (S. 47-64). (Zeitschrift für Bibliothekswesen und Bibliographie, Sonderband 111). Frankfurt am Main: Klostermann.

Haubfleisch, D. (2014). Die Bibliothek ist für ihre Nutzer da: Der Einfluss der nordrheinwestfälischen Gesamthochschulbibliotheken auf die Entwicklung des Fachreferats. In I. Siebert \& T. Lemanski (Hrsg.), Bibliothekare zwischen Verwaltung und Wissenschaft: 200 Jahre Berufsbilddebatte (S. 99-133). (Zeitschrift für Bibliothekswesen und Bibliographie, Sonderband 111). Frankfurt am Main: Klostermann.

IG WBS - Interessengruppe Wissenschaftliche BibliothekarInnen Schweiz IG WBS (2013). Berufsbild Wissenschaftliche Bibliothekarin / Wissenschaftlicher Bibliothekar. http://www. igwbs.ch/wp-content/uploads/igwbs_berufsbild_dt0901_26052015.pdf (17.04.2016).

IG WBS - Interessengruppe der Wissenschaftlichen Bibliothekarinnen und Bibliothekare der Schweiz (1994). Rundbrief an die Mitglieder, (4), 25.2.1994. http://www.igwbs.ch/ wp-content/uploads/2013/01/Rundbrief_04.pdf (25.4.2016).

IG WBS - Interessengruppe der Wissenschaftlichen Bibliothekarinnen und Bibliothekare der Schweiz (1993b). Rundbrief an die Mitglieder, (3), 16.11.1993. http://www.igwbs.ch/ wp-content/uploads/2013/01/Rundbrief_03.pdf (25.4.2016).

IG WBS - Interessengruppe der Wissenschaftlichen Bibliothekarinnen und Bibliothekare der Schweiz (1993a). Rundbrief an die Mitglieder, (2), Juni 1993. http://www.igwbs.ch/ wp-content/uploads/2013/01/Rundbrief_02.pdf (25.4.2016).

IG WBS - Interessengruppe der Wissenschaftlichen Bibliothekarinnen und Bibliothekare der Schweiz (1992). Rundbrief an die Mitglieder, (1), 22.11.1992. http://www.igwbs.ch/ wp-content/uploads/2013/01/Rundbrief_01.pdf (25.4.2016).

Ingold, M. (2015). Neue Tätigkeitsfelder und Ausbildungsmöglichkeiten in der Schweiz: Impulse und Perspektiven für das Berufsbild wissenschaftlicher Bibliothekarinnen und Bibliothekare. Vortrag, gehalten auf dem 104. Deutschen Bibliothekartag in Nürnberg vom 27.5.2015. urn:nbn:de:0290-opus4-19094

Jochum, U. (2014). Wissenschaftliche Bibliothekare. Ein Rettungsversuch. In I. Siebert \& T. Lemanski (Hrsg.), Bibliothekare zwischen Verwaltung und Wissenschaft: 200 Jahre Berufsbilddebatte (S. 135-145). (Zeitschrift für Bibliothekswesen und Bibliographie, Sonderband 111). Frankfurt am Main: Klostermann.

Munzinger Online/Duden - Deutsches Universalwörterbuch, 8. Aufl. (2015). „Berufsbild“. http:// www.munzinger.de/search/document?index=duden-d0\&id=D000000206\&type=text/ html\&query.key=uW5GSIMe\&template=/publikationen $/$ duden $/$ document. jsp\#D00000018734 (19.04.2016).

Oeggerli, E. \& Steinegger, U. (1995). Wie weiblich ist die Zukunft? Zum Bild und zur Situation von Frauen in der Bibliotheksarbeit. In: Für alle(s) offen. Bibliotheken auf neuen Wegen: 
Festschrift für Dr. Fredy Gröbli, Direktor der Oeffentlichen Bibliothek der Universität Basel (S. 198-208). Basel: Öffentliche Bibliothek der Universität Basel.

Oehling, H. (1998). Wissenschaftlicher Bibliothekar 2000 - quo vadis? 12 Thesen zur Zukunft des Fachreferenten. Bibliotheksdienst 32(2), S. 247-254.

Plappert, R. (2015). Einleitung zum Themenschwerpunkt „Berufsbild wissenschaftliche/r Bibliothekar/in“. o-bib - das offene Bibliotheksjournal, 2(3), 1-3. http://dx.doi. org/10.5282/o-bib/2015H3S1-3

Schaub, S. (2014a). Berufsbild Wissenschaftliche(r) Bibliothekar(in) heute - Anforderungen und Perspektiven (Frankfurt/Main, 30. September 2014). Info WBS 02. http://www. igwbs.ch/berufsbild-wissenschaftlicher-bibliothekarin-heute-anforderungen-undperspektiven-frankfurt-a-m-30-september-2014/ (17.04.2016).

Schaub, S. (2014b): Das neue Berufsbild für die wissenschaftlichen Bibliothekarlnnen der Schweiz. b.i.t.online 17(1), S. 32-35.

Schaub, S. \& Gavillet, G. (2014). Das neue Berufsbild „Wissenschaftliche Bibliothekarin / Wissenschaftlicher Bibliothekar": Le nouveau profil professionel „Bibliothécaire scientifique“. Vortrag, gehalten auf dem Kongress BIS in Lugano am 5. September 2014. http://www.igwbs.ch/wp-content/uploads/Berufsbild-Schaub-Gavillet.pdf (22.04.2016). Schibel, W. (1998). „Fachreferat 2000“: 13 Thesen zur Differenzierung des wissenschaftlichen Bibliotheksdienstes. Bibliotheksdienst 32(6), S. 1040-1047.

Senser, C. (1991). Die Bibliotheken der Schweiz. (Elemente des Buch- und Bibliothekswesens, Band 13). Wiesbaden: Reichert.

Siebert, I. (2014). Zur Renaissance des Wissenschaftlichen Bibliothekars. In I. Siebert \& T. Lemanski (Hrsg.), Bibliothekare zwischen Verwaltung und Wissenschaft: 200 Jahre Berufsbilddebatte (S. 25-46). (Zeitschrift für Bibliothekswesen und Bibliographie, Sonderband 111). Frankfurt am Main: Klostermann.

Siebert, I. \& Lemanski, T. (Hrsg.) (2014). Bibliothekare zwischen Verwaltung und Wissenschaft. 200 Jahre Berufsbilddebatte. (Zeitschrift für Bibliothekswesen und Bibliographie, Sonderband 111). Frankfurt/Main: Klostermann.

Söllner, K. (2014). Qualifikationswege und Berufsfelder in Bibliotheken. In R. Griebel, H. Schäffler \& K. Söllner (Hrsg.), Praxishandbuch Bibliotheksmanagement (S. 887-910). Berlin: De Gruyter Saur. DOI: 10.1515/9783110303261.887

Statistik Austria (2011). ISCO 08: Gemeinsame deutschsprachige Titel und Erläuterungen auf Basis der englischsprachigen Version 1.5a von April 2011. http://www.statistik.at/wcm/ idc/idcplg?IdcService=GET_PDF_FILE\&dDocName=049974 (17.04.2016).

Statistik der Bundesagentur für Arbeit (Hrsg.) (2016). Klassifikation der Berufe 2010 - alphabetisches Verzeichnis der Berufsbenennungen. https://statistik.arbeitsagentur.de/Statischer-Content/Grundlagen/Klassifikation-der-Berufe/KIdB2010/ Systematik-Verzeichnisse/Generische-Publikationen/Alphabetisches-Verzeichnis-Berufsbenennungen-Stand21012016.xls (17.04.2016).

Statistik der Bundesagentur für Arbeit (Hrsg.) (2013). Klassifikation der Berufe 2010 - Systematisches Verzeichnis. https://statistik.arbeitsagentur.de/Navigation/Statistik/Grundlagen/ Klassifikation-der-Berufe/KldB2010/KldB2010-Nav.html (17.04.2016).

SUPSI (La Scuola universitaria professionale della Svizzera italiana) \& Sbt (Sistema bibliotecario ticinese) (2012/2013). Library and Information Science: Master of Advanced Studies. http://www3.ti.ch/DECS/sw/temi/scuoladecs/attualita/allegati/ pdf-attualita-scuoladecs-70798590256.pdf (17.04.2016). 
Te Boekhorst, P., Buch, H. \& Ceynowa, K. (1998). „Wissenschaftlicher“ Bibliothekar 2000 - Hic Rhodus, hic salta! Bemerkungen zu Helmut Oehlings Thesen zur Zukunft des Fachreferenten. Bibliotheksdienst 32(4), S. 686-693.

VDB - Verein Deutscher Bibliothekarinnen und Bibliothekare (2014). Position des VDB zur Qualifikation als wissenschaftliche Bibliothekarin / wissenschaftlicher Bibliothekar. http:// www.vdb-online.org/wordpress/wp-content/uploads/2014/04/Position-des-VDB-zurQualifikation-als-wissenschaftliche_r-Bibliothekar_in-Final_18.03.2014.pdf (29.06.2016).

Wikipedia (2016a). „Beruf“. Version vom 2. Februar 2016. https://de.wikipedia.org/w/index. php?title=Beruf\&oldid=150985528 (17.04.2016).

\section{Weiterführende Literatur und Internetquellen}

BDB - Bundesvereinigung Deutscher Bibliotheksverbände: Arbeitsgruppe Gemeinsames Berufsbild (Hrsg.) (2000). Berufsbild 2000 - Bibliotheken und Bibliothekare im Wandel (2., unveränd. Nachdr. der dt. Fassung, erg. um die engl. Version). Wolfenbüttel: BDB.

BIB - Berufsverband Information Bibliothek e. V. - Kommission Ausbildung und Berufsbilder (2011). Berufsbild Wissenschaftliche/r Bibliothekar/in: Wir bringen Wissen in Bewegung: Berufsfeld Bibliothek und Information (3. leicht überarb. Aufl.). http://www.bib-info.de/ fileadmin/media/Dokumente/Berufsfeld/Berufsbild-Flyer.pdf (02.05.2016).

Höft, R. \& Holste-Flinspach, K. (2016). Karriere ohne Studium. Fachwirtausbildung in Hessen und Nordrhein-Westfalen bietet FaMls Möglichkeit zur beruflichen Weiterbildung. BuB Forum Bibliothek und Information, 68(4), S. 198-201.

VDB - Verein Deutscher Bibliothekarinnen und Bibliothekare (2015a). Thesen zum Round Table-Gespräch des VDB am 4. März - Teil 1. http://www.vdb-online.org/ wordpress/2015/02/24/thesen-zum-round-table-gesprach-des-vdb-am-4-marz-teil-1/ (29.6.2016).

VDB - Verein Deutscher Bibliothekarinnen und Bibliothekare (2015b). Thesen zum Round Table-Gespräch des VDB am 4. März - Teil 2. http://www.vdb-online.org/ wordpress/2015/03/01/thesen-zum-round-table-gesprach-des-vdb-am-4-marz-teil-2/ (29.06.2016).

VDB Bayern - Verein Deutscher Bibliothekarinnen und Bibliothekare: Landesverband Bayern (2014). Fortbildungsveranstaltung „Berufsbild Wissenschaftliche(r) Bibliothekar(in) heute - Anforderungen und Perspektiven “ am 30.9.2014, Dokumentation. http://www. vdb-online.org/veranstaltungen/657/ (29.06.2016).

VDB Sachsen - Verein Deutscher Bibliothekarinnen und Bibliothekare: Regionalverband Sachsen, Sachsen-Anhalt, Thüringen (2014). Stellungnahme zur Position des VDB zur Qualifikation als Wissenschaftliche Bibliothekarin / Wissenschaftlicher Bibliothekar. https://www.db-thueringen.de/servlets/MCRFileNodeServlet/dbt_derivate_00029824/ stellungnahme_\%20berufsbild_entwurf_final.pdf (29.06.2016).

Wikipedia (2016b). „Berufsbild“. Version vom 15. März 2016. https://de.wikipedia.org/w/index. php?title=Berufsbild\&oldid=152530690 (17.04.2016). 